This article was published as Smart Materials and Structures 28 (3), 035018. DOI: https://doi.org/10.1088/1361$\underline{665 X / a a f e a c}$ 


\title{
Magnetorheology of Alginate Ferrogels
}

\author{
Cristina Gila-Vilchez ${ }^{1}$, Juan D.G. Duran ${ }^{1}$, Fernando Gonzalez-Caballero ${ }^{1}$, Andrey \\ Zubarev $^{2,3,{ }^{*}}$ and Modesto T. Lopez-Lopez ${ }^{1,{ }^{*}}$ \\ ${ }^{1}$ Department of Applied Physics, University of Granada, Granada, Spain \\ ${ }^{2}$ Department of Theoretical and Mathematical Physics, Ural Federal University, Ekaterinburg, Russia \\ ${ }^{3}$ M.N. Mikheev Institute of Metal Physics, Ural Branch of the Russian Academy of Sciences, \\ Ekaterinburg, Russia
}

E-mail: a.j.zubarev@urfu.ru (AZ); modesto@ugr.es (MTLL)

Received xxxxxx

Accepted for publication $\mathrm{xxxxxx}$

Published xxxxxx

\begin{abstract}
Magnetorheological (MR) effect is a phenomenon typical of suspensions of magnetizable particles in a liquid carrier, characterized by strong changes of their mechanical properties in response to applied magnetic fields. Its origin is on the migration of magnetized particles and their aggregation into chain-like structures. However, for ferrogels, consisting of dispersions of magnetic particles in a polymer matrix, migration of particles is hindered by the elastic forces of the polymer network, preventing from strong MR effect. Interestingly, we demonstrate in this manuscript that strong MR effect in robustly cross-linked polymer ferrogels is still possible. Experimental results showed enhancement of the storage modulus of more than one order of magnitude for alginate ferrogels containing less than about $10 \mathrm{vol} \%$ of iron particles under moderate magnetic fields. The differential feature of these ferrogels is that, instead of individual particles, the disperse phase consisted of large clusters of iron microparticles homogeneously distributed within the polymer networks. These clusters of magnetic particles were formed at the stage of the preparation of the ferrogels and their presence within the polymer networks had two main consequences. First, the volume fraction of clusters was considerably larger than this of individual particles, resulting in a larger effective volume fraction of solids. Second, since the force of magnetic attraction between magnetic bodies is roughly proportional to the cube of the body size, the existence of such clusters favored inter-cluster interaction under a magnetic field and the appearance of strong MR effect. On this basis, we demonstrated by theoretical modeling that the strong MR effect displayed by the alginate ferrogels of the present work can be quantitatively explained by assuming the existence of large, roughly spherical particle aggregates formed at the stage of the preparation of the ferrogels. Our theoretical model provides a reasonable quantitative prediction of the experimental results.
\end{abstract}

Keywords: ferrogels, magnetorheology, storage modulus, magnetic particle

\section{Introduction}

Hydrogels can be defined as three-dimensional networks of flexible polymer chains capable of retaining large amounts of water. From the fundamental viewpoint, hydrogels are unique systems that combine large porosity and a mechanical behavior typical of an elastic solid. As a consequence, provided an adequate biocompatibility, hydrogels mimic the extracellular matrix (ECM) of living tissues more than any other kind of synthetic biomaterial [1]. Therefore, many biomedical applications have been conceived for hydrogels, some of them already exploited at a commercial level [1, 2]. For example, hydrogels have been used as artificial muscles $[3,4]$, reservoirs for storage and delivery of cells, drugs or environmental factors in clinical medicine $[5,6]$, as well as artificial ECM in tissue engineering [7].

In spite of the elevate resemblance of synthetic hydrogels to the ECM of living tissues, these biomaterials still present some obstacles that hinder a full development of their potential applications at a practical level. Among these drawbacks of synthetic hydrogels, the lack of adequate internal microstructure (e.g., large porosity and hierarchical, 
usually anisotropic, structure) stands out. Note that an adequate internal microstructure is not only a key factor for the attachment and proliferation of cells [8], but it is also essential for the adequate mechanical performance of biomaterials intended for the regeneration of hierarchically (from the nanoscopic to the macroscopic level) anisotropic tissues, such as for example skin, cartilage and muscles [9]. To tackle these limitations of conventional hydrogels and to gain a control over their microstuctural order, smart gels capable of modifying their properties in a permanent or temporary way in response to external stimuli (e.g., temperature, $\mathrm{pH}$, enzymes, etc.) are being developed [10].

An important category among these smart gels is constituted by magnetic gels (or ferrogels), which from the structural viewpoint consist of dispersions of magnetic microor nanoparticles embedded within a polymer gel. Recent reviews on the preparation and biomedical applications of ferrogels, and on their mechanical properties are given respectively in Ref. [11] and Ref. [12]. The main advantage of ferrogels against nonmagnetic hydrogels is that they can be actively manipulated and controlled by noncontact magnetic forces, changing their microstructure, as well as their macroscopic properties (shape, mechanical behavior, etc.) [13]. For example, several studies have demonstrated controlled (on-demand) release of drugs and cells from ferrogels upon tuning the intensity of an applied magnetic field [14-18]. Within the field of tissue engineering, several authors have also exploited magnetic field actuation, for example to assemble in a controlled manner microscale building blocks into complex tissue constructs [19], or to organize engineered cell tissues [20]. Also within the same field, we used fibrin polymer-based ferrogels as scaffolds for the generation of artificial magnetic tissues with mechanical properties tuneable by noncontact magnetic forces [21-23]. Furthermore, we recently demonstrated that the microstructure of fibrin hydrogels was completely reorganized by the presence of tiny amounts of magnetic nanoparticles within the hydrogel [24].

Tunability of the mechanical properties of dispersions of magnetizable particles by magnetic field application, a property known as magnetorheological (MR) effect [25], directly depends on the capability of the magnetic particles to migrate and aggregate into chain-like structures upon magnetic field application. In the case of ferrogels, the magnetic field-induced migration and aggregation of magnetic particles is partially hindered by the elastic forces of the polymer network, and consequently the MR effect developed by ferrogels is usually weak, as compared with suspensions of magnetic particles in a liquid carrier (or MR fluids). There are nevertheless some exceptions of ferrogels exhibiting "giant" MR effect, such as the carrageenan-based magnetic hydrogels reported in Ref. [26], or the alginate-based ferrogels reported in one of our recent works [27]. Note that this previous work of Ref. [27] was mainly devoted to the synthesis and rheological characterization of alginate ferrogels without magnetic field application, including only some preliminary results of their MR behavior. In the case of the carrageenan-based magnetic hydrogels of Ref. [26], the authors demonstrated that the magnetic particles indeed moved and formed long chain-like structures aligned in the direction of magnetic fields. In the present work, we thoroughly investigate from an experimental viewpoint the MR behavior of the alginate ferrogels and analyze the mechanisms behind the observed giant MR effect in these systems. Furthermore, we present a theoretical model that explains the observed MR effect. As it will be shown, the strong MR effect in alginate ferrogels is caused by the interaction under a magnetic field of preexisting clusters of magnetic particles within the alginate polymer network. Note also that, contrarily to the case of MR fluids where particle clusters should be avoided since they lead to quick phase separation under gravitational forces, the presence of particle clusters in ferrogels does not represent a nuisance because the polymer network confers colloidal stability against settling.

\section{Materials and Methods}

\subsection{Preparation of the ferrogels}

As magnetic phase we used silica-coated iron powders (commercial name: Fe-CC powder, supplied by BASF, Germany). These powders consisted of spherical iron particles, coated by a thin silica layer, having a total diameter of $1.4 \pm 0.6 \mu \mathrm{m}$ (as obtained by SEM pictures), volumetric mass density of $7.71 \pm 0.19 \mathrm{~g} \cdot \mathrm{cm}^{-3}$ (as obtained by a pycnometer), and a typical paramagnetic behavior with saturation magnetization $\mathrm{M}_{\mathrm{S}}=1587 \pm 2 \mathrm{kA} / \mathrm{m}$ (as obtained by SQUID magnetometry). As source of alginate polymer chains we used sodium alginate (supplied by Sigma Aldrich, USA), with empirical formula $\left(\mathrm{C}_{6} \mathrm{H}_{7} \mathrm{NaO}_{6}\right)_{\mathrm{n}}$, and molecular weight of $176.10 \mathrm{~g} / \mathrm{mol}$.

We prepared isotropic alginate ferrogels, consisting of FeCC particles embedded within a water-swollen alginate polymer network, by following the two-step protocol first reported in our previous work [27]. Briefly, we prepared a $1 \%$ $\mathrm{w} / \mathrm{v}$ solution of sodium alginate in distilled water. Then, for each $5 \mathrm{~mL}$ of solution of sodium alginate, we added $7.5 \mathrm{mg}$ of $\mathrm{CaCO}_{3}$ and $26.7 \mathrm{mg}$ of D-glucono- $\delta$-lactone (GDL), both supplied by Sigma Aldrich, USA, and homogenized the resulting mixture using a vortex mixer. Afterwards we placed the homogeneous mixture in a petri dish, and left it undisturbed under water-saturated atmosphere at room temperature. Note that GDL hydrolyzes in water to gluconic acid, enhancing the solubility of $\mathrm{CaCO}_{3}$; dissolved $\mathrm{CaCO}_{3}$ molecules act as source of $\mathrm{Ca}^{2+}$ ions, which provoke the crosslinking of alginate molecules by electrostatic bonding of each $\mathrm{Ca}^{2+}$ ion to two different alginate chains (each with valence - 
1), resulting in a three-dimensional (3-D) polymer network After 90 minutes the mixture was already jellified into a very soft alginate gel. At this moment we introduced the magnetic particles within the composition. For this aim we broke the alginate gel with the help of a vortex mixer, adding afterward the desired amount of magnetic particles. Finally we homogenized the resulting particle-alginate-water mixtures by 10 minutes of ultrasonication. After homogenization, given volumes of the mixtures were placed in petri dishes and we added to each of them the same volume of a $45 \mathrm{mM}$ solution of $\mathrm{CaCl}_{2}$ (provided by Sigma Aldrich, USA) in water. $\mathrm{CaCl}_{2}$ is a highly soluble source of $\mathrm{Ca}^{2+}$ ions that rapidly turned the homogeneous mixtures into homogeneous alginate ferrogels. Nevertheless, we left them overnight under water-saturated atmosphere at room temperature before magnetorheological characterization. An overview of the different ferrogels synthesized is shown in Table 1.

Table 1. Volume fraction of the different ferrogels

\begin{tabular}{|l|l|l|l|l|l|l|l|}
\hline Ferrogel & FG1 & FG2 & FG3 & FG4 & FG5 & FG6 & FG7 \\
\hline $\begin{array}{l}\text { Particle } \\
\text { conc. }\end{array}$ & 1.5 & 2.5 & 3.5 & 4 & 5 & 7.5 & 10 \\
vol $\%$ & vol $\%$ & vol $\%$ & vol $\%$ & vol $\%$ & vol $\%$ & vol $\%$ \\
\hline
\end{tabular}

\subsection{Microstructure of the ferrogels}

For the investigation of the microstructure of the ferrogels we used both optical microscopy connected to a CCD camara, and environmental scanning electron microscopy (ESEM), which was performed using a FEI QuemScan 650F ESEM.

\subsection{Magnetorheological characterization of the ferrogels}

For the characterization of the magnetorheological properties of the alginate ferrogels, we used a rotational magnetorheometer (Physica MCR 300) provided with a plateplate geometry of $20 \mathrm{~mm}$ of diameter. We studied the magnetorheological properties under shear of the ferrogels at different volume fractions of magnetic particles and at a constant temperature of $25 \pm 0.1^{\circ} \mathrm{C}$. Firstly, we determined the extension of the linear viscoelastic region (LVR) of the ferrogels under different values of the applied magnetic field. For this aim, we subjected the samples to oscillatory shear strain of constant frequency $(1 \mathrm{~Hz})$ and increasing amplitude (i.e., amplitude sweep tests) and monitored the values of the stroage $\left(G^{\prime}\right)$ and loss $\left(G^{\prime \prime}\right)$ moduli as a function of the shear strain amplitude. As will be discussed below (subsetion 3.2), from these experiments we could infer that a shear strain amplitude of $0.03 \%$ was well within the LVR.

Then, we subjected the samples to oscillatory shear strain of constant frequency of $1 \mathrm{~Hz}$ and fixed shear strain amplitude of $0.03 \%$ (corresponding to the LVR), and monitored the resulting response of the ferrogels for increasing magnetic field intensity in the range 0 to $282 \mathrm{kA} / \mathrm{m}$ (magnetic field sweep tests). During the experiments, we imposed a constant normal force of $0.1 \mathrm{~N}$ in order to ensure that there was always contact between the upper plate of the rheometer and the ferrogels. As a reference, note the range of shear forces applied during rheological measurements: $6 \cdot 10^{-4}-0.2 \mathrm{~N}$.

In order to obtain statistically significant results, we measured 3 different samples for each set of experimental conditions. In this paper we show the corresponding mean values and standard deviations.

\section{Experimental results}

\subsection{Microstructure of the ferrogels}

Macroscopic observation as well as low resolution optical microscopy demonstrated homogeneous ferrogels in all cases. A closer view of the ferrogels by means of high resolution optical microscopy revealed the existence of a disperse phase, seen as dark spots, homogeneously dispersed within the polymer network (Figure 1a). ESEM images confirmed that in all cases this disperse phase consisted of large clusters of magnetic particles, instead of individual particles (Figure 1c).

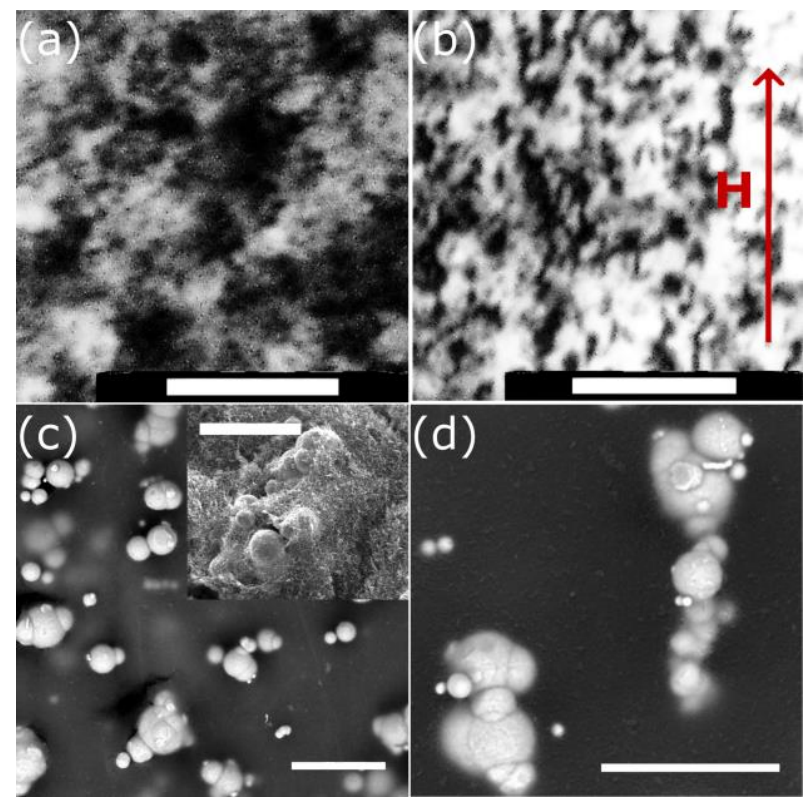

Fig.1. Microscopy images of ferrogels. (a, c) Pristine ferrogels, never subjected to applied magnetic field. (b, d) Ferrogels previously subjected to an applied magnetic field of approx. 280 $\mathrm{kA} / \mathrm{m}$ (direction is indicated) -note that the field was switched off before microscopic observations. Optical microscopy was used in

$(\mathbf{a}, \mathbf{b})$, while ESEM microscopy in (c, d). Volume fraction of magnetic particles was 0.3 vol\% in all cases. Bar length: $100 \mu \mathrm{m}$ (a, b) and $10 \mu \mathrm{m}(\mathbf{c}, \mathbf{d})$. Inset in (c) was taken by a different technique to visualize the polymer fibers as well as the magnetic particles

The question arising at this point, and before analysing the magnetorheological properties of the ferrogels is whether these particle clusters were able to move and aggregate into chain-like structures in response to an applied magnetic field. Direct observation of the process of rearrangement of the 
clusters in response of an applied magnetic field by optical microscopy was impossible because of the impracticality of in situ combination of strong enough magnetic field with optical microscopy. Nevertheless, we confirmed that application of strong enough magnetic fields to the completely cross-linked ferrogels induced the formation of permanent chain-like secondary aggregates that remained even after switching off the field (Figures $1 \mathrm{~b}$ and 1d). Consequently, we can conclude that particle clusters were able to move in response to an applied magnetic field.
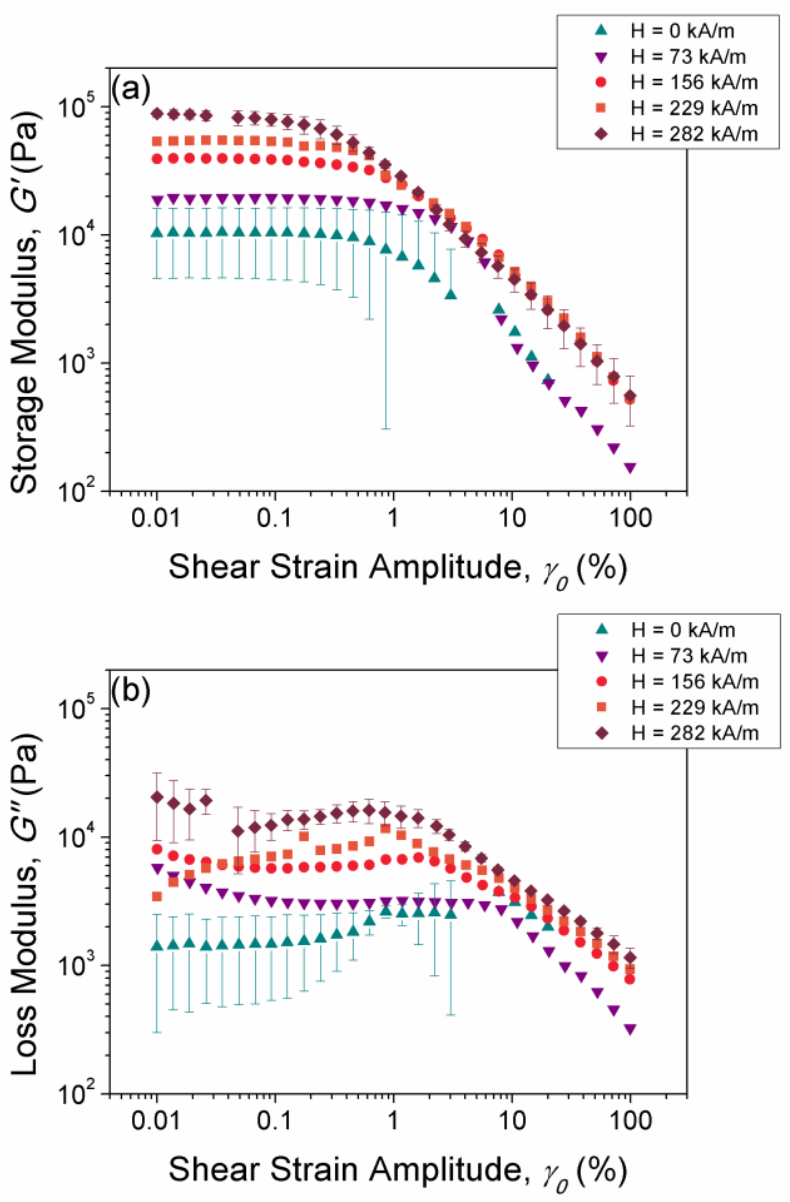

Fig.2. (a) Storage modulus and (b) loss modulus of magnetic hydrogels containing $5 \mathrm{vol} \%$ volume fraction of magnetic particles as functions of the strain amplitude for oscillatory measurements at a fixed frequency of $1 \mathrm{~Hz}$. Magnetic field strengths are indicated.

Note that the same axis scale is used in both parts

\subsection{Magnetorheological characterization of the} ferrogels

Amplitude sweep tests demonstrated that the ferrogels presented a trend typical of cross-linked systems, with approximately constant values of $G^{\prime}>G^{\prime \prime}$ at low shear strain amplitude, followed by a decrease of both magnitudes as the shear strain amplitude was increased above $0.2-2 \%$-see as an example results for 5 vol.\% of magnetic particles in Figure 2. Note that the initial part (low values of shear strain amplitude) for which $G^{\prime}$ and $G^{\prime \prime}$ are approximately constant is identified with the LVR.

Results of magnetic field sweep tests demonstrate an increase of the storage modulus with the intensity of the magnetic field strength for all the ferrogels under study (Fig. 3 ). As mentioned in the introduction, this dependence of the rheological properties on the intensity of the magnetic field is known as MR effect, and it has its origin in the polarization of magnetizable particles and their subsequent attraction and aggregation into elongated structures under the influence of a magnetic field [25]. Strong MR effect is typical for suspensions of particles in a liquid-like medium, known as MR fluids or MR suspensions. When the particles are dispersed in an elastic medium, e.g., a polymer gel or elastomer, their magnetic field-induced migration and aggregation is partially hindered by the elastic forces of the polymer network [28]. As a consequence, the MR effect developed by magnetic gels and elastomers is weaker (usually much weaker) than this developed by MR fluids [29-31]. Nevertheless, as observed in Fig.3, our alginate ferrogels show strong MR effect, especially at the highest volume fractions under study $(7.5 \%$ and $10 \%)$, in spite of the relatively robust polymer network. This strong MR effect in our ferrogels should be a consequence of the existence of particle aggregates even in the absence of applied magnetic field, as discussed below in section 4 . In fact, the force of magnetic attraction between particles (aggregates) is roughly proportional to the cube of the particle (aggregate) size, whereas the force of the elastic resistance to the particle displacement linearly depends on this size. Therefore, the balance of magnetic forces to elastic forces is more favorable to migration in the case of relatively large aggregates as compared with individual particles. Furthermore, the migration would be easier the higher the applied magnetic field, which justify the faster-than-linear enhancement of the storage modulus with the magnetic field observed in Fig. 3a at low to medium magnetic field intensity. Note that at the highest field intensities, the increase of the storage modulus is approximately linear, very likely as a consequence of the tendency to magnetic saturation of the particles. 

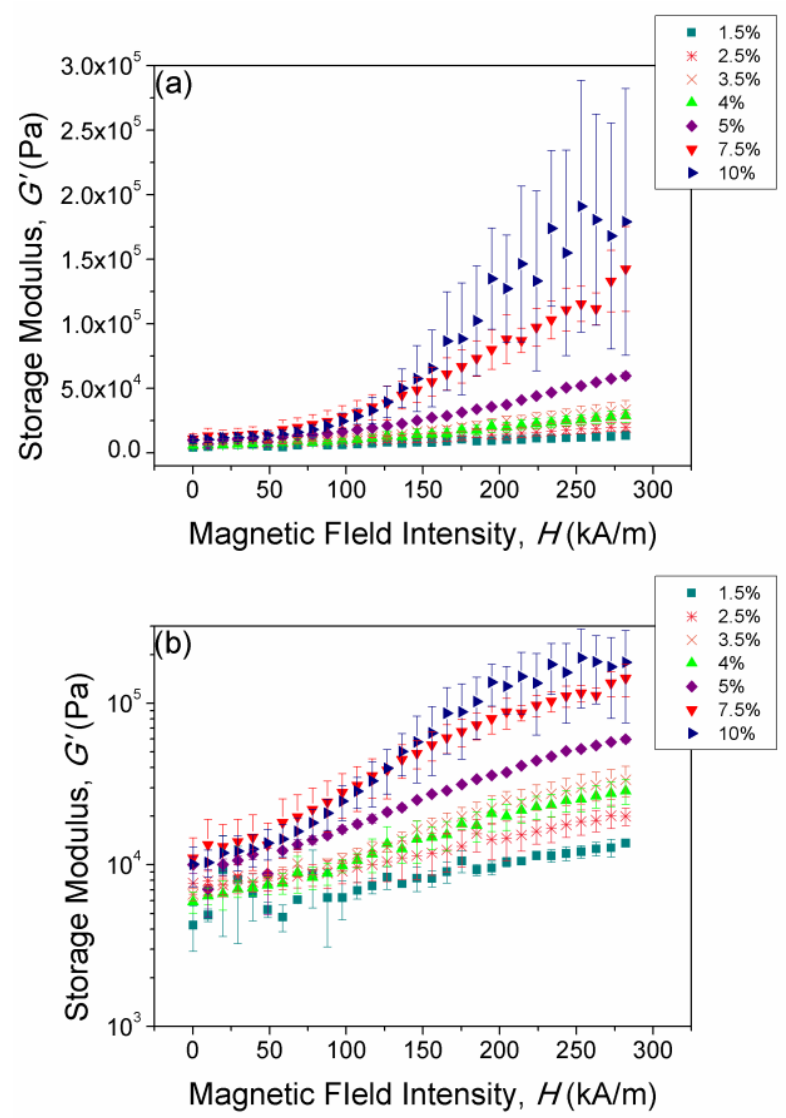

Fig.3. Storage modulus as a function of the magnetic field strength for different alginate ferrogels. (a): $y$-axis in linear scale; (b): $y$-axis in logarithmic scale. The volume fraction of magnetic particles within the ferrogels is indicated.

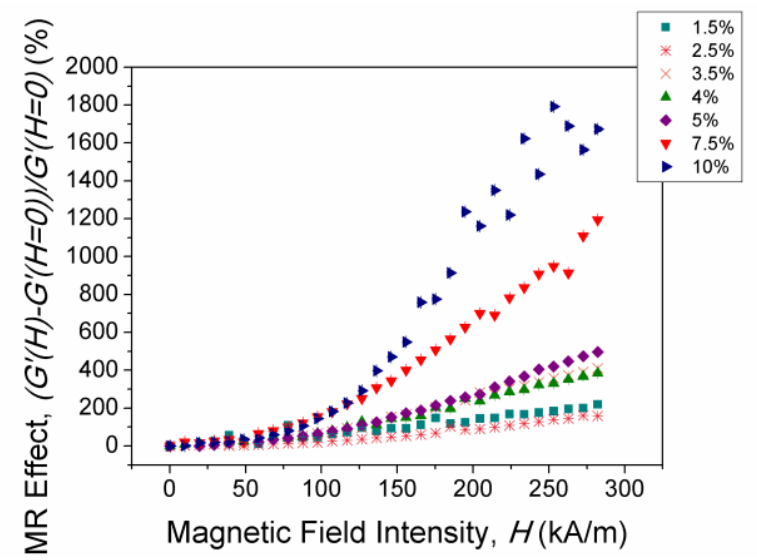

Fig.4. Magnetorheological (MR) effect, quantified as the relative increase of the storage modulus under a magnetic field, $G^{\prime}(H)$, with respect to the storage modulus in the absence of field, $G^{\prime}(H=$ $0)$, for different alginate ferrogels. The volume fraction of magnetic particles within the ferrogels is indicated.

In addition, as the volume fraction of magnetic particles increases, we expect that the volume fraction of initial aggregates will be higher and thus they will be closer to each other. As a consequence, it is expected that the MR effect becomes stronger as the volume fraction of magnetic particles within the ferrogel increases, something that in fact is in agreement with experimental results. This stronger MR effect as the volume fraction of magnetic particles increases is best seen if we plot the relative increase of the storage modulus under a field, $G^{\prime}(H)$, with respect to the storage modulus in the absence of field, $G^{\prime}(H=0)$ - see Fig.4. As an example, note that according to Fig.4, the MR effect for the ferrogel containing $10 \%$ of magnetic particles at $250 \mathrm{kA} / \mathrm{m}$ is about $1600 \%$, whereas under the same applied magnetic field, the MR effect for the ferrogel containing only $1.5 \%$ of magnetic particles is only of about $50 \%$.

\section{Theoretical model and comparison with experiments}

Electron microscopy observations (see Fig.3 in [27] and Fig. 1 here) show that without external magnetic field, particles, embedded in alginate ferrogel, form dense isotropic clusters (primary agglomerates). Possibly, this clusterisation takes place because of specific adhesion interaction between the particles and the host polymer at the stage preceding its curing. Discussion of the similar effect one can find in [32].

At the same time experiments of [27] demonstrate very strong dependence of the alginate ferrogel shear storage modulus on the volume fraction of the particles; the measured dependence appears much stronger than the traditional theories of composite materials, based on the concept of separate particles, predict. Accounting appearance of these agglomerates has allowed us to explain quantitatively the observed strong volume fraction effect in the absence of the field [27]. That is why here we will consider the alginate ferrogels as composites, consisting of the elastic host polymer network and clusters (primary agglomerates) composed of magnetic particles. For maximal simplification of the mathematical part of the problem, we will suppose that the isotropic clusters are identical spheres. Of course, this is a very strong approximation; however it allows us to get estimates of the MR effect, at least at a semi-quantitative level. The model of the systems is illustrated in Fig.5. Note that simplification of the primary agglomerates as indentical spheres was successfully used in the model of [27] of rheological properties of the alginate ferrogels out of the field. 


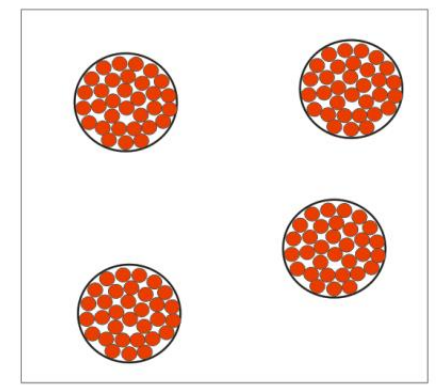

Fig. 5. Sketch of the primary agglomerates in the alginate ferrogel

We suppose that, under the action of the applied magnetic field, the spherical primary agglomerates are magnetized and unite into elongated "secondary" chain-like structures, as illustrated in Fig.6.

It should be noted that field induced aggregation of single magnetizable particles, embedded in elastic media, have been detected in experiments [33-35] as well as in computer simulations [36]. In ref. [37] hysteretic pair aggregation of magnetizable particles in an elastic medium has been studied theoretically.

The typical size of these chains is determined by competition between the force of magnetic attraction between the primary aggregates and elastic forces in the host polymer.

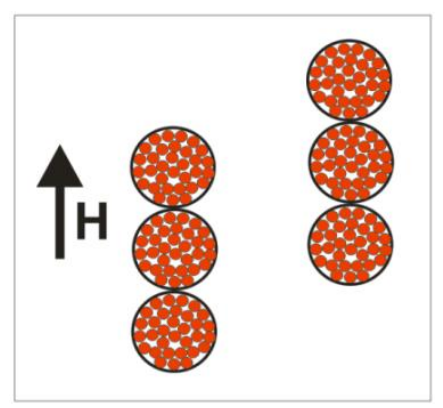

Fig. 6. Sketch of the composite with the chain-like "secondary" aggregates

For maximal simplicity, we will suppose that the chains are identical. Now, under the action of the magnetic field, we can consider the ferrogel as a composite consisting of the elastic matrix and identical elongated magnetizable chains. Let us denote the aspect ratio of this chain (i.e., the number of primary agglomerates in the chain) as $r$. We will estimate this number by using the hierarchical model of the chain formation in a soft elastic medium suggested in Ref. [38]. The details of the model reported in Ref. [38] are cumbersome and are consequently omitted here.

Following to the general theory of mechanics of polar (magnetizable) media (see, for example, Refs. [39-42]), one can present the storage modulus of the composite as:

$$
\mathrm{G}^{\prime}=\mathrm{G}_{\mathrm{s}}^{\prime}+\mathrm{G}_{\mathrm{a}}^{\prime}
$$

The modulus $\mathrm{G}_{\mathrm{s}}$ corresponds to the symmetrical stress due to elastic deformations in the host matrix. The part $\mathrm{G}_{\mathrm{a}}^{\prime}$ corresponds to the antisymmetrical stress, provided by magnetic torques, acting on the chains. By using the identity of the Navier-Stokes equation at low Reynolds number and the Lamé equation of linear deformations of elastic media, as well as the Krieger-Dougherty (KD) formula [43] for the effective viscosity of suspensions, we can estimate $G_{S}^{\prime}$ as:

$$
\mathrm{G}_{\mathrm{s}}^{\prime}=\mathrm{G}_{0}^{\prime}\left(1-\Phi_{\mathrm{agg}} / \Phi_{\mathrm{m}}\right)^{-\left[\mathrm{G}^{\prime}\right] \Phi_{\mathrm{m}}}
$$

Here $G^{\prime}{ }_{0}$ is the modulus of the host polymer, $\Phi_{\text {agg }}$ is the volume fraction of the aggregates, $\Phi_{\mathrm{m}}$ is the volume fraction of the dense packing (we estimate $\Phi_{\mathrm{m}} \approx 0.64$ ), the magnitude $\left[G^{\prime}\right]$ is determined from the condition that the complex $\mathrm{G}^{\prime}{ }_{0}\left(1+\left[G^{\prime}\right] \Phi_{\text {agg }}\right)$ is equal to the modulus of extremely diluted $\left(\Phi_{\text {agg }} \rightarrow 0\right)$ composite. It is worthy to mention at this point that although KD relation is an empirical expression, it has been found correct for the prediction of the viscosity and viscoelastic moduli of suspensions in general [44], and in particular for the predicition of the viscoelastic moduli of alginate ferrogels [27].

For the spherical aggregates, shown in Fig.1, the Einstein relation $\left[G^{\prime}\right]=2.5$ holds. In order to estimate $\left[G^{\prime}\right]$ for the chainlike aggregates, illustrated in Fig.6, we will model the chains as ellipsoids of revolution. For the dilute suspensions of the parallel ellipsoids with the aspect ratio $r$, one can use the following relation [42]:

$$
\begin{gathered}
\mathrm{G}_{\mathrm{s}}^{\prime}=\mathrm{G}_{0}^{\prime}(1+[\alpha(\mathrm{r})+0.5(\mathrm{~s}(\mathrm{r})+\beta(\mathrm{r}) \lambda(\mathrm{r})+\beta(\mathrm{r})) \\
\left.+\chi(\mathrm{r})-2 \beta(\mathrm{r}) \lambda(\mathrm{r})] \Phi_{\mathrm{agg}}\right)
\end{gathered}
$$

Thus, for chains:

$$
\begin{aligned}
{\left[\mathrm{G}^{\prime}\right]=\alpha(\mathrm{r})+0.5 } & (s(\mathrm{r})+\beta(\mathrm{r}) \lambda(\mathrm{r})+\beta(\mathrm{r}))+\chi(\mathrm{r}) \\
& -2 \beta(\mathrm{r}) \lambda(\mathrm{r})
\end{aligned}
$$

Expressions for the functions appearing in equation (4) are given in the Appendix. Note that for spherical aggregates $(r=1) \alpha=2.5, \beta=\zeta=\lambda=\chi=0$, i.e., the Einstein relation $\left[G^{\prime}\right]=2.5$ holds. Note that equations (3) and (4) hold for dilute systems. Nevertheless, Batchelor [45] showed that in the case of highly elongated inclusions (particle chains in our case), the effect of the individual inclusions (chains) dominates over the effects of inter-inclusion (inter-chain) interaction, which justifies the use of equations (3) and (4) for all the volume fractions under study.

Let us consider the dilute enough composite with the whole volume fraction of the embedded particles $\Phi=0.048$. For this 
composite without applied magnetic field experiments give $G^{\prime} \approx 10200 \mathrm{~Pa}$. Taking into account that $\mathrm{G}_{0}^{\prime}=4800 \mathrm{~Pa}$, we estimate $\Phi_{\mathrm{agg}} \approx 0.25$. And from this estimation we can estimate the volume fraction of particles in the aggregate:

$$
\varphi=\frac{\Phi}{\Phi_{\mathrm{agg}}} \approx 0.2
$$

In order to estimate $G^{\prime}$, let us consider the chain-like aggregate, deviated because of the macroscopic shear of the gel, from the direction of the applied magnetic field (Fig.7).

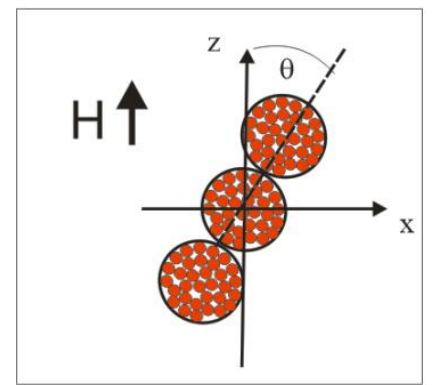

Fig. 7. Sketch of the chain-like aggregate, deviated from the direction of the magnetic field by the shear

Following [42], we can estimate the modulus $\mathrm{G}^{\prime}{ }_{\mathrm{a}}$ as:

$$
\mathrm{G}_{\mathrm{a}}^{\prime}=\frac{\mu_{0}}{2} \Phi_{\mathrm{agg}} \frac{\left(\chi_{\mathrm{agg}}\right)^{2}\left(\mathrm{~N}_{\perp}(\mathrm{r})-\mathrm{N}_{\|}(\mathrm{r})\right)}{\left(1+\chi_{\mathrm{agg}} \mathrm{N}_{\|}(\mathrm{r})\right)\left(1+\chi_{\mathrm{agg}} \mathrm{N}_{\perp}(\mathrm{r})\right)} \mathrm{H}^{2}
$$

Here $\mu_{0}$ is the magnetic permeability of vacuum, $\chi_{\text {agg }}$ is the magnetic susceptibility of the chain-like aggregate, $\mathrm{N}_{\|}(\mathrm{r})$ and $\mathrm{N}_{\perp}(\mathrm{r})$ are the demagnetizing factors of the chainlike aggregates along and perpendicular to their major axis of symmetry. Again, modeling the chain-like aggregates as ellipsoids of revolution, we get (see, for example, Ref. [39]):

$$
\mathrm{N}_{\|}=\frac{1}{2\left(r^{2}-1\right)^{\frac{3}{2}}}\left[r \ln \left(\frac{r+\sqrt{r^{2}-1}}{r-\sqrt{r^{2}-1}}\right)-2 \sqrt{r^{2}-1}\right], \mathrm{N}_{\perp}=\frac{1-\mathrm{N}_{\|}}{2}
$$

Now, we will take into account that the particle magnetization nonlinearly depends on the field $H$. Let us denote the internal field inside the chain as $H_{i n}$. Since only small shear deformations are considered here, it is reasonable to suppose that the angle $\theta$ of the chain axis deviation from the field $H$ is also small. In this case the internal field can be estimated as [39]:

$$
\mathrm{H}_{\text {in }}=\mathrm{H}-\mathrm{N}_{\|} \mathrm{M}_{\text {in }}
$$

Here $M_{\text {in }}$ is the magnetization inside the chain. In order to estimate $M_{i n}$, we will use the Fröhlich-Kennelly relation [46]:

$$
M_{\text {in }}=\frac{\chi_{0} H_{i n} M_{s}}{\chi_{0} H_{\text {in }}+M_{s}}
$$

Here $\chi_{0}$ is the initial susceptibility of the chain and $\mathrm{M}_{\mathrm{s}}$ is its saturation magnetization.

Combining eqs. (8) and (9), we come to a square equation for $\mathrm{M}_{\mathrm{in}}$. Its solution is:

$$
\begin{gathered}
M_{\text {in }}=\frac{b-\sqrt{b^{2}-4 \chi_{0}^{2} M_{s} H N_{\|}}}{2 N_{\|} \chi_{0}} \\
b=\chi_{0} H+M_{s}\left(1+\chi_{0} N_{\|}\right)
\end{gathered}
$$

By definition the aggregate susceptibility is:

$$
\chi_{\text {agg }}=\frac{M_{\text {in }}}{H_{\text {in }}}=\frac{M_{\text {in }}}{H-N_{\|} M_{\text {in }}}
$$

The initial susceptibility $\chi_{0}$ and the saturation magnetization $M_{s}$ of the primary agglomerates cannot be calculated theoretically, because they are determined by the details of the stochastic internal morphology of the particles in ) the aggregates. Because of this, we have estimated these values as $\chi_{0}=6.4, M_{s}=800 \mathrm{kA} / \mathrm{m}$ by fitting the results of calculations of $G^{\prime}$ to the experimental ones at particle volume fraction $4.8 \%$, and then we used them for all other volume fractions of the particles. Taking into account the values $\chi_{0} \approx 6.4 ; \mathrm{M}_{\mathrm{s}} \approx 800 \mathrm{kA} / \mathrm{m}$ and the aggregate volume fraction $\Phi_{\mathrm{agg}}=0.25$, as well as $\mathrm{G}_{0}{ }_{0}=4800 \mathrm{~Pa}$; by using the models of chains formations in soft elastic matrixes given in Ref. [38], we finally can calculate the number of primary agglomerates in the chains, which is equal to the chain aspect ratio $r$. The results are shown in Fig.8. Note that the calculated $r$ (Fig.8) must be interpreted as the typical or averaged aspect ratio over all real chains, which must of course present some size distribution. With respect to the shape of the curve in Fig.8, it is explained by the dependence of the chain magnetization, $M$, on the applied magnetic field, $H$. At low values of $H, M$ increases linearly with $H$, which leads to the observed approximate square dependence of $r$ on $H$. At higher values of $H, M$ reaches saturation and, as a consequence, a weaker, approximately linear dependence, of $r$ on $H$ is obtained. 


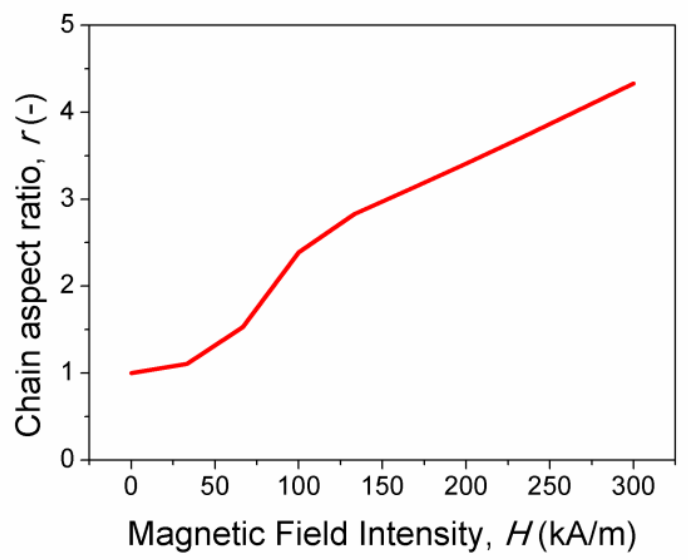

Fig.8. Chain aspect ratio $r$ (number of primary spherical agglomerates in the chain) as a function of the magnetic field $H$ $(\mathrm{kA} / \mathrm{m})$ for $4.8 \mathrm{vol} . \%$ of particles

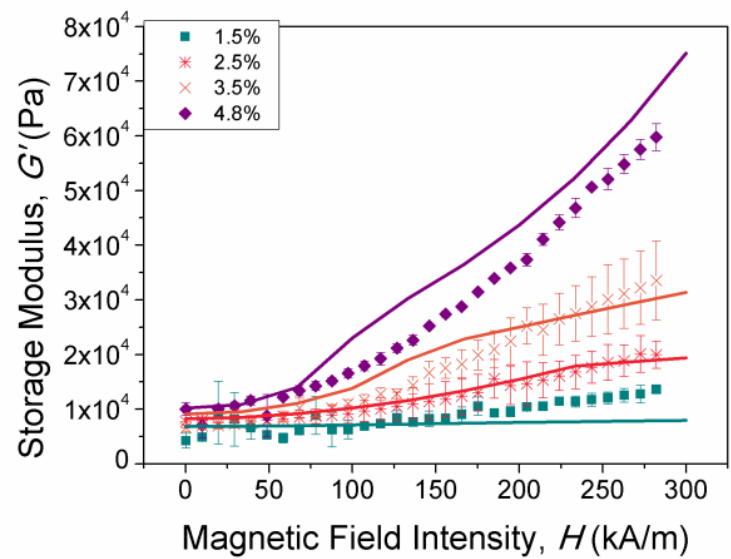

Fig.9. Storage modulus as a function of the magnetic field intensity for different alginate ferrogels. Symbols represent experimental results and lines the predictions of the theoretical model.

Some results of comparison of the theoretical calculations of the modulus $G^{\prime}$ with our experiments are presented in Figs. 9 and 10. In order to make the calculations for all particles volume fractions $\Phi$, presented in Figs. 9 and 10, we used the approach described in this section for the volume fraction $\Phi=4.8 \%$. Namely, first, by using eqs. (2) and (4), we calculated $G^{\prime}$ in the absence of magnetic field $(r=1$; $\alpha=2.5, \beta=\zeta=\lambda=\chi=0$ ) as a function of the aggregate volume fraction $\Phi_{\text {agg }}$. Then, by comparison of the calculated results with the corresponding measured values of this modulus, we estimated the volume fraction $\Phi_{\text {agg }}$ in the form of function of the particle volume fraction $\Phi$. Afterwards, by using the approach of Ref. [38], we determined the dependence of the aspect ratio $r$ on the field $H$ for each volume fraction $\Phi_{\text {agg }}$, therefore, for each value of $\Phi$. Finally, we calculated the modulus $G^{\prime}$ by using eq. (2). For small volume fractions (Fig. 9) the model is in good agreement with the experimental results, which could be taken as a proof of the validity of the model and the estimates required for calculations for this experimental situation. An exception is the understimation of the growth of the storage modulus with the magnetic field intensity (more evident at the highest values of the field) for particle volume fraction $1.5 \%$. On the contrary, for volume fraction $7.5 \%$ (see Fig. 10) and higher (not shown here), the disagreement is very strong (more than 3 times of overestimation).

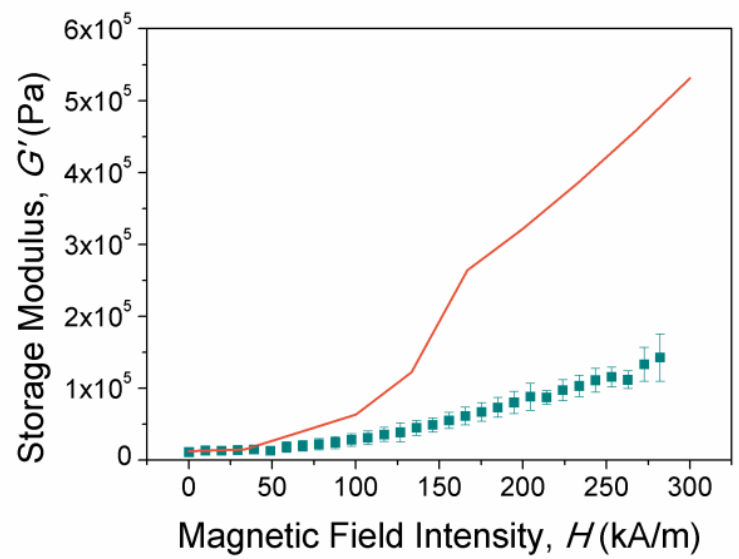

Fig.10. Storage modulus ( $\square$ ) as a function of the magnetic field intensity for magnetic ferrogels with particle volume fraction 7.5 vol.\%. Line represents theoretical model predictions. Disagreement between experiments and theory for volume fraction $10 \mathrm{vol} . \%$ (not shown here) is even worse.

The strong disagreement at high fields between theoretical and experimental results, evidenced in Fig. 10, can be explained by the simplicity of our consideration of unification of primary agglomerates into separate chains. When volume fraction increases, this assumption becomes worse. For example, according to our estimated, when the particle volume fraction $\Phi$ is $7.5 \%$, the volume fraction of the agglomerates $\Phi_{\text {agg }}$ is about $29 \%$. At this high volume fraction of agglomerates, the real agglomerates should have irregular shape and can interpenetrate, which can affect significantly the final results. In addition, for the sake of simplicity, we have not taken into account the effects of the sample demagnetizing field and the fact that the field inside the sample is smaller than the applied field. This cannot lead to serious mistakes when the particle volume fraction $\Phi$ is low enough (as in the case of results in Fig. 9). However, at high enough volume fraction the mistake can be significant. Finally, at high volume fractions of the agglomerates, $\Phi_{\text {agg }}$, the existence of separate chains in the ferrogels should not be hypothesized. One can rather expect the appearance of more topologically complicated thick structures with smaller aspect ratio $r$ [47].

Let us finally mention that although the theoretical model could be applied, with similar results, to ferrogels consisting of larger spherical particles, instead of clusters of smaller 
particles, from the experimental point of view, the use of smaller particles that aggregate into clusters is preferred for several reasons, including the following. (i) The dispersion of smaller particles to get homogeneous ferrogels is easier than in the case of larger particles; (ii) clusters of smaller particles present a much higher surface than spherical particles of equal volume, and consequently they can interact more effectively with the polymer molecules; (iii) furthermore, as evidenced by SEM observations (Figure 1c-inset in this manuscript, as well as Figure 3 in Ref. 27) the clusters are not only constituted by particles, but also by polymer molecules. As a consequence of all these reasons, smaller particles that aggregate into clusters integrate much better within the polymer network, and are thus preferred.

\section{Conclusions}

We have reported a comprehensive investigation on the MR properties of alginate ferrogels, consisting of clusters of iron microparticles homogeneously dispersed in alginate hydrogels. Our experimental results demonstrate that these alginate ferrogels exhibit strong MR effect, in spite of the expected hindrance of particle movement by elastic forces of the polymer network. As an example, for a ferrogel containing $10 \mathrm{vol} \%$ of magnetic particles, the increase of the storage modulus under a field of $250 \mathrm{kA} / \mathrm{m}$ with respect to the modulus in the absence of field reaches about $1600 \%$. We have found that particle clusters formed at the stage of the preparation of the ferrogels may be behind the unexpectedly strong MR effect. In fact, inside an elastic medium such a hydrogel, particle clusters will be more prone to magnetic field-induced chaining than individually isolated particles, since the force of magnetic attraction is roughly proportional to the cube of the size of the cluster (particle), whereas the force of the elastic resistance to the cluster (particle) displacement linearly depends on this size. Furthermore, since the volume fraction of clusters is larger than the volume fraction of particles, this results in clusters being on average closer to each other than isolated particles, something that also favors magnetic attraction between particles and thus chaining. We have presented a model based on these hypotheses. Despite the simplifications (spherical shape and identical size of the primary agglomerates; identical size of the chains), this provides a good quantitative prediction of the dependence of the storage modulus of ferrogels with the magnetic field strength at low volume fraction of magnetic particles. At particle volume fractions above 5 vol.\%, our model strongly overestimates the experimental results, as a likely consequence of the used simplifications.

To conclude, our work presents a convenient way for the preparation of homogeneous ferrogels with strong MR effect: agglomeration of magnetic particles into clusters at the preparation stage of ferrogels. Since particle clusters do not lead to gravitational settling in ferrogels due to their embedment into the polymer network, this approach may be used to enhance the controllability of ferrogels and ferroelastomers in technical and biomedical applications that require good control of their properties by applied magnetic fields, such as smart dampers or artificial muscles. Finally, note that alginate hydrogels, because of their abundance in source, biocompatibility and ease of handling, have found broad popularity in several fields such as biotechnology, food industry and pharmaceutical industry [48], which makes even more relevant the results of our work.

\section{Acknowledgements}

This study was supported by project FIS2017-85954-R (Ministerio de Economía, Industria y Competitividad, MINECO, and Agencia Estatal de Investigación, AEI, Spain, cofunded by Fondo Europeo de Desarrollo Regional, FEDER, European Union). CGV acknowledges financial support by Ministerio de Ciencia, Innovación y Universidades and University of Granada, Spain, for her FPU17/00491 grant. AZ is grateful to the Program of the Ministry of Education and Science of the Russian Federation, projects 02.A03.21.0006, 3.1438.2017/4.6, and 3.5214.2017/6.7 and the Russian Fund of Basic Researches, project 18-08-00178.

\section{Appendix}

Functions appearing in equation (4) can be expressed as it follows [42]:

$$
\begin{aligned}
\alpha & =\frac{1}{r \alpha_{0}^{\prime}} \\
\beta & =\frac{2\left(r^{2}-1\right)}{r\left(r^{2} \alpha_{0}+\beta_{0}\right)} \\
\zeta & =\frac{4}{r \beta_{0}^{\prime}\left(r^{2}+1\right)}-\frac{2}{r \alpha_{0}^{\prime}} \\
\chi & =\frac{2 \alpha_{0}^{\prime \prime}}{r \alpha_{0}^{\prime} \beta_{0}^{\prime \prime}}-\frac{8}{r \beta_{0}^{\prime}\left(r^{2}+1\right)}+\frac{2}{r \alpha_{0}^{\prime}} \\
\lambda_{n} & =\frac{r^{2}-1}{r^{2}+1} \\
\text { and } & -\frac{1}{r^{2}-1}\left[\frac{2}{r}+\frac{1}{\sqrt{r^{2}-1}} \ln \left(2 r^{2}-1-2 r \sqrt{r^{2}-1}\right)\right] \\
\alpha_{0} & =\frac{1}{\beta^{2}-1}\left[r-\frac{1}{2 \sqrt{r^{2}-1}} \ln \left(2 r^{2}-1+2 r \sqrt{r^{2}-1}\right)\right] \\
\alpha_{0}^{\prime} & =\frac{1}{4\left(r^{2}-1\right)^{2}}\left[r\left(2 r^{2}-5\right)\right. \\
2 \sqrt{r^{2}-1} & \left.\ln \left(2 r^{2}-1-2 n \sqrt{r^{2}-1}\right)\right]
\end{aligned}
$$

$$
\begin{aligned}
\beta_{0}^{\prime}=\frac{1}{\left(n^{2}-1\right)^{2}}[ & \frac{n^{2}+2}{n} \\
& \left.-\frac{3}{2 \sqrt{n^{2}-1}} \ln \left(2 n^{2}-1+2 n \sqrt{n^{2}-1}\right)\right]
\end{aligned}
$$




$$
\begin{aligned}
\alpha_{0}^{\prime \prime}=\frac{1}{4\left(r^{2}-1\right)^{2}}[ & r\left(2 r^{2}+1\right) \\
& \left.-\frac{4 r^{2}-1}{2 \sqrt{r^{2}-1}} \ln \left(2 r^{2}-1+2 r \sqrt{r^{2}-1}\right)\right] \\
\beta_{0}^{\prime \prime}=-\frac{1}{\left(r^{2}-1\right)^{2}} & {[3 r} \\
& \left.+\frac{2 r^{2}+1}{2 \sqrt{r^{2}-1}} \ln \left(2 r^{2}-1-2 r \sqrt{r^{2}-1}\right)\right]
\end{aligned}
$$

\section{References}

[1] Caló E and Khutoryanskiy VV 2015 Biomedical applications of hydrogels: A review of patents and commercial products Eur. Polym. J. 65 252-267

[2] Gahawar AK, Peppas NA and Khademhosseini A 2014 Nanocomposite Hydrogels for Biomedical Applications Biotechnology and Bioengineering 111 441-453

[3] Ismail YA, Martínez JG, Al Harrasi AS, Kim SJ and Otero TF 2011 Sensing characteristics of a conducting polymer/hydrogel hybrid microfiber artificial muscle Sensors and Actuators B $\mathbf{1 6 0}$ 1180-1190

[4] Tondu B, Emirkhanian R, Mathé S and Ricard A 2009 A pHactivated artificial muscle using the McKibben- type braided structure Sensors and Actuators A 150 124-130

[5] Park KM, Lee SY, Joung YK, Na JS, Lee MC and Park KD 2009 Thermosensitive chitosan-Pluronic hydrogel as an injectablecell delivery carrier for cartilage regeneration Acta Biomaterialia 5 1956-1965

[6] Yang B, Zhang Y, Zhang X, Tao L, Li S and Wei Y 2012 Facilely prepared inexpensive and biocompatible self-healing hydrogel: a new injectable cell therapy carrier Plym. Chem. 3 3235-3238

[7] Tibbitt MW and Anseth KS 2009 Hydrogels as Extracellular Matrix Mimics for 3D Cell Culture Biotechnology and Bioengineering 103 655-663

[8] Murphy CM, Haugh MG and O'Brien FJ 2010 The effect of mean pore size on cell attachment, proliferation and migration in collagen-glycosaminoglycan scaffolds for bone tissue engineering Biomaterials 31 461-466

[9] Moutos FT, Freed LE and Guilak F 2007 A biomimetic threedimensional woven composite scaffold for functional tissue engineering of cartilage Nature Materials 6 162-167

[10] Ebara M, Kotsuchibashi Y, Narain R, Idota N, Kim YJ, Hoffman JM, Uto K and Aoyagi T 2014 Smart Biomaterials (Tokyo: Japan, Springer, pp 9-65)

[11] Li Y, Huang G, Zhang X, Li B, Chen Y, Lu T, Lu TJ and Xu F 2012 Magnetic Hydrogels and Their Potential Biomedical Applications Adv. Funct. Mater. 23 660-672

[12] Lopez-Lopez MT, Durán JDG, Iskakova LY and Zubarev AY 2016 Mechanics of magnetopolymer composites: a review $J$. Nanofluids 5 479-495

[13] Thévenot J, Oliveira H, Sandre O and Lecommandoux S 2013 Magnetic responsive polymer composite materials Chem. Soc. Rev. 42 7099-7116

[14] Zhao X, Kim J, Cezar CA, Huebsch N, Lee K, Bouhadir K and Mooney DJ 2011 Active scaffolds for on-demand drug and cell delivery PNAS 108 67-72
[15] Hu SH, Liu TY, Liu DM and Chen SY 2007 Controlled pulsatile drug release from a ferrogel by a high-frequency magnetic field Macromolecules 40 6786-6788

[16] Hu SH, Chen SY, Liu DM and Hsiao CS 2008 Core/singlecrystal-shell nanospheres for controlled drug release via a magnetically triggered rupturing mechanism Adv Mater 20 2690-2695

[17] Liu TY, Hu SH, Liu TY, Liu DM and Chen SY 2006 Magnetic-sensitive behavior of intelligent ferrogels for controlled release of drug Langmuir 22 5974-5978

[18] Hu SH, Liu TY, Liu DM and Chen SY 2007 Nanoferrosponges for controlled drug release $J$ Controlled Release 121 181-189

[19] Xu F, Wu CM, Rengarajan V, Finley TD, Keles HO, Sung Y, Li B, Gurkan UA and Demirci U 2011 Three-Dimensional Magnetic Assembly of Microscale Hydrogels Adv. Mater. 23 4254-4260

[20] Grogan SP, D'Lima DD, Colwell CW and Jin S 2012 In situ tissue engineering using magnetically guided three dimensional cell patterning US patent US2012214217 A1

[21] Lopez-Lopez MT, Scionti G, Oliveira AC, Duran JDG, Campos A, Alaminos M and Rodriguez IA 2015 Generation and Characterization of Novel Magnetic Field-Responsive Biomaterials PLOS ONE 10 1-17

[22] López-López MT, Durán JDG, Alaminos M, Rodriguez IA and Scionti G 2016 Elaboración de tejidos artificiales que comprenden partículas magnéticas World patent WO/2016/079366

[23] Rodriguez-Arco L, Rodriguez IA, Carriel V, BonhomeEspinosa AB, Campos F, Kuzhir P, Duran JDG and Lopez-Lopez MT 2016 Biocompatible magnetic core-shell nanocomposites for engineered magnetic tissues Nanoscale $\mathbf{8} 8138-8150$

[24] Bonhome-Espinosa AB, Campos F, Rodriguez IA, Carriel V, Marins JA, Zubarev A, Duran JDG and Lopez-Lopez MT 2017 Effect of particle concentration on the microstructural and macromechanical properties of biocompatible magnetic hydrogels Soft Matter 13 2928-2941

[25] Bossis G, Volkova O, Lacis S and Meunier A 2002 Magnetorheology: Fluids, Structures and Rheology LNP 594 202-230

[26] Mitsumata T, Honda A, Kanazawa H and Kawai M 2012 Magnetically tunable elasticity for magnetic hydrogels consisting of carrageenan and carbonyl iron particles J. Phys. Chem. B 116 12341-12348

[27] Gila-Vilchez C, Bonhome-Espinosa AB, Kuzhir P, Zubarev A, Duran JDG and Lopez-Lopez MT 2018 Rheology of magnetic alginate hydrogels J. Rheol. 62 1083-1096

[28] Biller AM, Stolbov OV and Raikher YL 2014 Modeling of particle interactions in magnetorheological elastomers J. Appl. Phys. 116114904

[29] Abramchuk S, Kramarenko E, Stepanov G, Nikitin LV, Filipcsei G, Khokhlov AR and Zrinyi M 2007 Novel highly elastic magnetic materials for dampers and seals: part II. Material behavior in a magnetic field Polym. Adv. Technol. 18 513-518

[30] Nikitin LV, Stepanov GV, Mironova LS and Gorbunov AI 2004 Magnetodeformational effect and effect of shape memory in magnetoelastics J. Magn. Magn. Mater. 272-276 2072-2073 
[31] Varga Z, Filipcsei G and Zrinyi M 2006 Magnetic field sensitive functional elastomers with tuneable elastic modulus Polymer 47 227-233

[32] Rose S, Prevoteau A, Elziere P, Hourdet D, Marcellan D and Leibler L 2013 Nanoparticle solutions as adhesives for gels and biological tissues Nature $\mathbf{5 0 5} 382-385$

[33] Gundermann T, Cremer P, Löwen H, Menzel AM and Odenbach S 2017 Statistical analysis of magnetically soft particles in magnetorheological elastomers Smart Mater. Struct. 26045012

[34] Schümann M and Odenbach A 2017 In-situ observation of the particle microstructure of magnetorheological elastomers in presence of mechanical strain and magnetic fields Journal of Magnetism and Magnetic Materials 441 88-92

[35] Puljiz M, Huang S, Kalina KA, Nowak J, Odenbach S, Kästner M, Auernhammerb GK and Menzel AM 2018 Reversible magnetomechanical collapse: virtual touching and detachment of rigid inclusions in a soft elastic matrix Soft Matter 14 6809-6821

[36] Pessot G, Schümann M, Gundermann T, Odenbach S, Löwen H and Menzel AM 2018 Tunable dynamic moduli of magnetic elastomers: from characterization by $\mathrm{x}$-ray microcomputed tomography to mesoscopic modeling J. Phys.: Condens. Matter 30125101 (15pp)

[37] Biller AM, Stolbov OV and Raikher YL 2015 Mesoscopic magnetomechanical hysteresis in a magnetorheological elastomer Physical Review E 92023202

[38] Zubarev AY, Chirikov DN, Borin DY and Stepanov GV 2016 Hysteresis of the magnetic properties of soft magnetic gels SoftMatter 12 6473-6480

[39] Landau LD and Lifshitz EM 1960 Electrodynamics of Continuous Media (New York: USA, Pergamon Press)

[40] Rosensweig RE 1985Ferrohydrodynamics (New York: USA, Cambridge University Press)

[41] Doi M and Edwards SF 1986 The Theory of Polymer Dynamics (New York: USA, Oxford University Press)

[42] Pokrovskii VN 1978 Statistical Mechanics of Dilute Suspensions (Moscow: Russia, Nauka)

[43] Krieger IM and Dougherty TJ 1959 A mechanism for nonNewtonian flow in suspension of rigid spheres Trans. Soc. Rheol. 3 137-152

[44] Barnes HA, Hutton JF and Walters K 1989 An introduction to rheology (Amsterdam: Netherlands, Elsevier)

[45] Batchelor G 1971 The stress generated in a non-dilute suspension of elongated particles by pure straining motion $J$. Fluid Mech 46 813-829

[46] Bozorth R 1993 Ferromagnetism (New York: USA, Wiley)

[47] Dohmen E, Borin D and Zubarev A 2017 Magnetic field angle dependent hysteresis of a magnetorheological suspension Journal of Magnetism and Magnetic Materials 443 275-280

[48] Kuo CK and Ma PX 2001 Ionically crosslinked alginate hydrogels as scaffolds for tissue engineering: Part 1. Structure, gelation rate and mechanical properties. Biomaterials 22511 521 